\title{
Effect of Different Nutrient Levels on Growth and Yield of Browntop Millet
}

\author{
H. P. Kiran ${ }^{*}$ \\ Dept of Agronomy, GKVK, Bangalore, India \\ *Corresponding author
}

A B S T R A C T

\begin{tabular}{|l|}
\hline Ke y w or d s \\
Browntop millet, \\
Nutrient levels, \\
Yield, Economics \\
\hline Article Info \\
\hline $\begin{array}{l}\text { Accepted: } \\
20 \text { December } 2020 \\
\text { Available Online: } \\
\text { 10 January } 2021\end{array}$ \\
\hline
\end{tabular}

\section{Introduction}

Browntop millet (Brachiaria ramosa) is an annual warm-season grass and majorly used in forage management systems. Browntop millet is originated in Southeast Asia. It is grown in the regions of scanty and erratic rainfall, poor and marginal soils in southern India. It is popular as a short duration crop, higher yield per unit time, low input requirement, known for its drought and shade tolerance as it can withstand severe moisture stress and suited to wide range of soil conditions.

The Browntop millet can fill narrow growing windows to produce a good quality forage because of its extremely rapid growth. Under ideal conditions, seed will germinate within 56 days and forage will be ready to harvest within two months' time. The nutritional composition of Browntop millet is better when compared to other millets. The grain is a rich source of natural fibre (12.5 per cent), carbohydrate, protein (11.5 per cent), minerals (6.21 per cent), calcium (18 per cent) and iron (8.9 per cent). It is a food for patients suffering from diabetes.

Browntop millet is grown under adverse environmental conditions; and best suited under moisture stress deprived soils. However, in reality, the potential of this crop is not attained fully. Browntop millet being low nutrient demanding crops, but responds well for addition of N, P and K. Depleted soil 
nutrient status and cultivation of improved varieties in Browntop millet needs balanced fertilizer through external fertilizers. The exact requirement of major nutrients for Browntop millet is not worked out and established so far. As this millet is of short duration and has high canopy growth as compared to other millets, the present investigation is planned to find out the nitrogen, phosphorous and potassium requirement of Browntop millet and their interactions.

\section{Materials and Methods}

The experiment to study the performance of the crop to different fertilizer levels on growth and yield in Browntop millet consisted of fourteen treatments replicated thrice in a randomized complete block design (RCBD). This experiment was conducted during Kharif 2019 at Gandhi KrishiVignan Kendra (GKVK).

The soil is red sandy loam and the treatment tested were, $\mathrm{T}_{1}: 20: 20: 10 \mathrm{~kg} \mathrm{~N}: \mathrm{P}: \mathrm{K} \mathrm{ha}^{-1}, \mathrm{~T}_{2}$ : 20:20:20 kg N:P:K ha ${ }^{-1}, \mathrm{~T}_{3}: 20: 30: 10 \mathrm{~kg}$ $\mathrm{N}: \mathrm{P}: \mathrm{K} \mathrm{ha}^{-1}, \mathrm{~T}_{4}:$ 20:30:20 kg N:P:K ha ${ }^{-1}, \mathrm{~T}_{5}$ : 40:20:10 kg N:P:K ha ${ }^{-1}, \mathrm{~T}_{6}: 40: 20: 20 \mathrm{~kg}$ $\mathrm{N}: \mathrm{P}: \mathrm{K} \mathrm{ha}^{-1}, \mathrm{~T}_{7}:$ 40:30:10 kg N:P:K ha ${ }^{-1}, \mathrm{~T}_{8}$ : 40:30:20 kg N:P:K ha ${ }^{-1}, \mathrm{~T}_{9}: 60: 20: 10 \mathrm{~kg}$ $\mathrm{N}: \mathrm{P}: \mathrm{K} \mathrm{ha}^{-1}, \mathrm{~T}_{10}: 60: 20: 20 \mathrm{~kg} \mathrm{~N}: \mathrm{P}: \mathrm{K} \mathrm{ha}^{-1}, \mathrm{~T}_{11}$ : 60:30:10 kg N:P:K ha ${ }^{-1}, \mathrm{~T}_{12}: 60: 30: 20 \mathrm{~kg}$ $\mathrm{N}: \mathrm{P}: \mathrm{K} \mathrm{ha}^{-1}, \mathrm{~T}_{13}: 40: 20: 0 \mathrm{~kg} \mathrm{~N}: \mathrm{P}: \mathrm{K} \mathrm{ha}^{-1}$ and $\mathrm{T}_{14}$ : Control.

Local variety (Dundukorale) was sown at the spacing of $45 \times 10 \mathrm{~cm}$. The gross and net plot sizes were $3.0 \times 4.5 \mathrm{~m}$ and $2.6 \times 2.7 \mathrm{~m}$ respectively. Data averaged over three replication and the data on Browntop millet growth parameter and yield parameter were collected.

The data collected on different traits was statistically analysed using the standard procedure and the results were tested at five percent level of significance as given by Gomez and Gomez (1984).

\section{Results and Discussion}

\section{Growth attributes}

At harvest, application of $60 \mathrm{~kg} \mathrm{~N} \mathrm{ha}^{-1}+30$ $\mathrm{kg} \mathrm{P}_{2} \mathrm{O}_{5} \mathrm{ha}^{-1}+20 \mathrm{~kg} \mathrm{~K}_{2} \mathrm{O} \mathrm{ha}^{-1}\left(\mathrm{~T}_{12}\right)$ significantly showed higher plant height $(110.33 \mathrm{~cm})$ compared to application of $40 \mathrm{~kg}$ $\mathrm{N} \mathrm{ha}{ }^{-1}+20 \mathrm{~kg} \mathrm{P}_{2} \mathrm{O}_{5} \mathrm{ha}^{-1}+0 \mathrm{~kg} \mathrm{~K}_{2} \mathrm{O} \mathrm{ha}^{-1}$ $(89.50 \mathrm{~cm})\left(\mathrm{T}_{13}\right)$ and control $(84.33 \mathrm{~cm})\left(\mathrm{T}_{14}\right)$. However, it was on par with application of 60 $\mathrm{kg} \mathrm{N} \mathrm{ha}^{-1}+30 \mathrm{~kg} \mathrm{P}_{2} \mathrm{O}_{5} \mathrm{ha}^{-1}+10 \mathrm{~kg} \mathrm{~K}_{2} \mathrm{O} \mathrm{ha}^{-1}$ $(102 \mathrm{~cm})\left(\mathrm{T}_{11}\right)$.

Leaf area of treatment $\left(\mathrm{T}_{12}\right)$ i.e., application of $60 \mathrm{~kg} \mathrm{~N} \mathrm{ha}^{-1}+30 \mathrm{~kg} \mathrm{P}_{2} \mathrm{O}_{5} \mathrm{ha}^{-1}+20 \mathrm{~kg} \mathrm{~K}_{2} \mathrm{O}$ $\mathrm{ha}^{-1}$ showedsignificantly higher leaf area $\left(755.57 \mathrm{~cm}^{2}\right)$ compared to application of 40 $\mathrm{kg} \mathrm{N} \mathrm{ha}{ }^{-1}+20 \mathrm{~kg} \mathrm{P}_{2} \mathrm{O}_{5} \mathrm{ha}^{-1}+0 \mathrm{~kg} \mathrm{~K}_{2} \mathrm{O}$ ha $^{-}$ ${ }^{1}\left(617.34 \mathrm{~cm}^{2}\right)\left(\mathrm{T}_{13}\right)$ and control $\left(559.15 \mathrm{~cm}^{2}\right)$ $\left(\mathrm{T}_{14}\right)$ at harvest.

Application of $60 \mathrm{~kg} \mathrm{~N} \mathrm{ha}^{-1}+30 \mathrm{~kg} \mathrm{P}_{2} \mathrm{O}_{5} \mathrm{ha}^{-1}$ $+20 \mathrm{~kg} \mathrm{~K}_{2} \mathrm{O}$ ha $^{-1}$ significantly showed higher dry matter $(9.55 \mathrm{~g}) \quad\left(\mathrm{T}_{12}\right)$ compared to application of $40 \mathrm{~kg} \mathrm{~N} \mathrm{ha}^{-1}+20 \mathrm{~kg} \mathrm{P}_{2} \mathrm{O}_{5} \mathrm{ha}^{-1}$ $+0 \mathrm{~kg} \mathrm{~K}_{2} \mathrm{O} \mathrm{ha}^{-1}$ (6.37) $\left(\mathrm{T}_{13}\right)$ and control (5.52 g) $\left(\mathrm{T}_{14}\right)$ at harvest.

This increased in growth parameter might be due to availability of sufficient nutrients to plants, which leads to anatomical changes such as increase in size of cells, intercellur spaces, thinner cell walls, lower development of epidermal tissue, better root growth, and better translocation of photosynthates resulting in vigorous plant growth resulted in favourable plant height, leaf area index, dry matter accumulation. Similar finding were reported by Nigade and More (2012), Lingegowda et al., (1986) in finger millet. 
Table.1 Plant height $(\mathrm{cm})$, Leaf area $\left(\mathrm{cm}^{2}\right)$ and Dry matter accumulation $(\mathrm{g})$ as influenced by different levels of major nutrients in Browntop millet

\begin{tabular}{|c|c|c|c|}
\hline Treatments & $\begin{array}{l}\text { Plant height } \\
\text { (cm) }\end{array}$ & $\begin{array}{l}\text { Leaf area } \\
\quad\left(\mathrm{cm}^{2}\right)\end{array}$ & $\begin{array}{c}\text { Dry matter } \\
\text { accumulation (g) }\end{array}$ \\
\hline $\mathrm{T}_{1}: 20: 20: 10 \mathrm{~N}: \mathrm{P}_{2} \mathrm{O}_{5}: \mathrm{K}_{2} \mathrm{O} \mathrm{kg} \mathrm{ha}^{-1}$ & 86.66 & 589.19 & 5.66 \\
\hline $\mathrm{T}_{2}: 20: 20: 20 \mathrm{~N}: \mathrm{P}_{2} \mathrm{O}_{5}: \mathrm{K}_{2} \mathrm{O} \mathrm{kg} \mathrm{ha}^{-1}$ & 87.96 & 594.18 & 6.01 \\
\hline$T_{3}: 20: 30: 10 \mathrm{~N}: \mathrm{P}_{2} \mathrm{O}_{5}: \mathrm{K}_{2} \mathrm{O} \mathrm{kg} \mathrm{ha}^{-1}$ & 89.90 & 600.87 & 6.33 \\
\hline$T_{4}: 20: 30: 20 \mathrm{~N}: \mathrm{P}_{2} \mathrm{O}_{5}: \mathrm{K}_{2} \mathrm{O} \mathrm{kg} \mathrm{ha}^{-1}$ & 93.66 & 657.47 & 6.61 \\
\hline $\mathrm{T}_{5}: 40: 20: 10 \mathrm{~N}: \mathrm{P}_{2} \mathrm{O}_{5}: \mathrm{K}_{2} \mathrm{O} \mathrm{kg} \mathrm{ha}^{-1}$ & 94.66 & 632.44 & 6.44 \\
\hline $\mathrm{T}_{6}: 40: 20: 20 \mathrm{~N}: \mathrm{P}_{2} \mathrm{O}_{5}: \mathrm{K}_{2} \mathrm{O} \mathrm{kg} \mathrm{ha}^{-1}$ & 93.33 & 637.88 & 6.48 \\
\hline $\mathrm{T}_{7}: 40: 30: 10 \mathrm{~N}: \mathrm{P}_{2} \mathrm{O}_{5}: \mathrm{K}_{2} \mathrm{O} \mathrm{kg} \mathrm{ha}^{-1}$ & 98.00 & 661.42 & 6.71 \\
\hline $\mathrm{T}_{8}: 40: 30: 20 \mathrm{~N}: \mathrm{P}_{2} \mathrm{O}_{5}: \mathrm{K}_{2} \mathrm{O} \mathrm{kg} \mathrm{ha}^{-1}$ & 100.60 & 673.90 & 7.35 \\
\hline$T_{9}: 60: 20: 10 \mathrm{~N}: \mathrm{P}_{2} \mathrm{O}_{5}: \mathrm{K}_{2} \mathrm{O} \mathrm{kg} \mathrm{ha}^{-1}$ & 99.40 & 665.48 & 6.80 \\
\hline$T_{10}: 60: 20: 20 \mathrm{~N}: \mathrm{P}_{2} \mathrm{O}_{5}: \mathrm{K}_{2} \mathrm{O} \mathrm{kg} \mathrm{ha}^{-1}$ & 100.66 & 681.37 & 7.44 \\
\hline $\mathrm{T}_{11}: 60: 30: 10 \mathrm{~N}: \mathrm{P}_{2} \mathrm{O}_{5}: \mathrm{K}_{2} \mathrm{O} \mathrm{kg} \mathrm{ha}^{-1}$ & 102.00 & 684.54 & 7.87 \\
\hline$T_{12}: 60: 30: 20 \mathrm{~N}: \mathrm{P}_{2} \mathrm{O}_{5}: \mathrm{K}_{2} \mathrm{O} \mathrm{kg} \mathrm{ha}^{-1}$ & 110.33 & 755.57 & 9.55 \\
\hline $\mathrm{T}_{13}: 40: 20: 0 \mathrm{~N}: \mathrm{P}_{2} \mathrm{O}_{5}: \mathrm{K}_{2} \mathrm{O} \mathrm{kg} \mathrm{ha}{ }^{-1}$ & 89.50 & 617.34 & 6.37 \\
\hline $\mathbf{T}_{14}$ : Control & 84.33 & 559.15 & 5.52 \\
\hline S. Em \pm & 2.98 & 22.49 & 0.54 \\
\hline CD at $5 \%$ & 9.05 & 68.24 & 1.64 \\
\hline
\end{tabular}

Table. 2 Productive tillers, panicle length, grain yield and straw yield as influenced by different levels of major nutrients in Browntop millet

\begin{tabular}{|c|c|c|c|c|}
\hline Treatments & $\begin{array}{l}\text { Productive } \\
\text { tillers }\end{array}$ & $\begin{array}{c}\text { Panicle } \\
\text { length } \\
\text { (cm) }\end{array}$ & $\begin{array}{c}\text { Grain yield } \\
\text { (kg/ha) }\end{array}$ & $\begin{array}{c}\text { Straw yield } \\
\text { (kg/ha) }\end{array}$ \\
\hline $\mathrm{T}_{1}: 20: 20: 10 \mathrm{~N}: \mathrm{P}_{2} \mathrm{O}_{5}: \mathrm{K}_{2} \mathrm{O} \mathrm{kg} \mathrm{ha}^{-1}$ & 9.36 & 16.00 & 841 & 1786 \\
\hline $\mathrm{T}_{2}: 20: 20: 20 \mathrm{~N}: \mathrm{P}_{2} \mathrm{O}_{5}: \mathrm{K}_{2} \mathrm{O} \mathrm{kg} \mathrm{ha}^{-1}$ & 9.46 & 16.42 & 850 & 1875 \\
\hline $\mathrm{T}_{3}: 20: 30: 10 \mathrm{~N}: \mathrm{P}_{2} \mathrm{O}_{5}: \mathrm{K}_{2} \mathrm{O} \mathrm{kg} \mathrm{ha}^{-1}$ & 10.00 & 16.48 & 873 & 1910 \\
\hline $\mathrm{T}_{4}: 20: 30: 20 \mathrm{~N}: \mathrm{P}_{2} \mathrm{O}_{5}: \mathrm{K}_{2} \mathrm{O} \mathrm{kg} \mathrm{ha}^{-1}$ & 10.50 & 16.88 & 918 & 2142 \\
\hline $\mathrm{T}_{5}: 40: 20: 10 \mathrm{~N}: \mathrm{P}_{2} \mathrm{O}_{5}: \mathrm{K}_{2} \mathrm{O} \mathrm{kg} \mathrm{ha}^{-1}$ & 10.30 & 16.83 & 911 & 2033 \\
\hline $\mathrm{T}_{6}: 40: 20: 20 \mathrm{~N}: \mathrm{P}_{2} \mathrm{O}_{5}: \mathrm{K}_{2} \mathrm{O} \mathrm{kg} \mathrm{ha}^{-1}$ & 10.46 & 16.83 & 914 & 2122 \\
\hline $\mathrm{T}_{7}: 40: 30: 10 \mathrm{~N}: \mathrm{P}_{2} \mathrm{O}_{5}: \mathrm{K}_{2} \mathrm{O} \mathrm{kg} \mathrm{ha}^{-1}$ & 10.60 & 16.9 & 934 & 2215 \\
\hline $\mathrm{T}_{8}: 40: 30: 20 \mathrm{~N}: \mathrm{P}_{2} \mathrm{O}_{5}: \mathrm{K}_{2} \mathrm{O} \mathrm{kg} \mathrm{ha}^{-1}$ & 10.86 & 17.14 & 1044 & 2488 \\
\hline $\mathrm{T}_{9}: 60: 20: 10 \mathrm{~N}: \mathrm{P}_{2} \mathrm{O}_{5}: \mathrm{K}_{2} \mathrm{O} \mathrm{kg} \mathrm{ha}^{-1}$ & 10.73 & 16.96 & 935 & 2370 \\
\hline $\mathrm{T}_{10}: 60: 20: 20 \mathrm{~N}: \mathrm{P}_{2} \mathrm{O}_{5}: \mathrm{K}_{2} \mathrm{O} \mathrm{kg} \mathrm{ha}^{-1}$ & 11.60 & 17.25 & 1066 & 2575 \\
\hline $\mathrm{T}_{11}: 60: 30: 10 \mathrm{~N}: \mathrm{P}_{2} \mathrm{O}_{5}: \mathrm{K}_{2} \mathrm{O} \mathrm{kg} \mathrm{ha}^{-1}$ & 11.86 & 18.15 & 1078 & 2799 \\
\hline $\mathrm{T}_{12}: 60: 30: 20 \mathrm{~N}: \mathrm{P}_{2} \mathrm{O}_{5}: \mathrm{K}_{2} \mathrm{O} \mathrm{kg} \mathrm{ha}^{-1}$ & 13.93 & 19.33 & 1295 & 3131 \\
\hline $\mathrm{T}_{13}: 40: 20: 0 \mathrm{~N}: \mathrm{P}_{2} \mathrm{O}_{5}: \mathrm{K}_{2} \mathrm{O} \mathrm{kg} \mathrm{ha}^{-1}$ & 10.20 & 16.49 & 903 & 1994 \\
\hline$T_{14}$ : Control & 9.33 & 15.71 & 762 & 1682 \\
\hline S. Em \pm & 0.61 & 0.50 & 65.85 & 146.28 \\
\hline CD at $5 \%$ & 1.85 & 1.51 & 199.75 & 443.76 \\
\hline
\end{tabular}




\section{Yield attributes}

Significantly, higher number of productive tillers per plant was noticed in the application of $60 \mathrm{~kg} \mathrm{~N} \mathrm{ha}{ }^{-1}+30 \mathrm{~kg} \mathrm{P}_{2} \mathrm{O}_{5} \mathrm{ha}^{-1}+20 \mathrm{~kg}$ $\mathrm{K}_{2} \mathrm{O}$ ha $^{-1}(13.93)\left(\mathrm{T}_{12}\right)$ compared to other levels of fertilizers. Whereas, the lower number of productive tillers were noticed in control $(9.33)\left(\mathrm{T}_{14}\right)$ followed by the application of $40 \mathrm{~kg} \mathrm{~N}^{-1}+20 \mathrm{~kg} \mathrm{P}_{2} \mathrm{O}_{5} \mathrm{ha}^{-1}+$ $0 \mathrm{~kg} \mathrm{~K}_{2} \mathrm{O} \mathrm{ha}^{-1}(10.20)\left(\mathrm{T}_{13}\right)$.

Ear head length differed significantly with the application of $60 \mathrm{~kg} \mathrm{~N} \mathrm{ha}^{-1}+30 \mathrm{~kg} \mathrm{P}_{2} \mathrm{O}_{5} \mathrm{ha}^{-1}$ $+20 \mathrm{~kg} \mathrm{~K}_{2} \mathrm{O} \mathrm{ha}^{-1}(19.33 \mathrm{~cm})\left(\mathrm{T}_{12}\right)$ compared to application of $40 \mathrm{~kg} \mathrm{~N} \mathrm{ha}^{-1}+20 \mathrm{~kg} \mathrm{P}_{2} \mathrm{O}_{5}$ $\mathrm{ha}^{-1}+0 \mathrm{~kg} \mathrm{~K}_{2} \mathrm{O} \mathrm{ha}^{-1}(16.49 \mathrm{~cm})\left(\mathrm{T}_{13}\right)$ and control $(15.71 \mathrm{~cm})\left(\mathrm{T}_{14}\right)$. However, it was found on par with application of $60 \mathrm{~kg} \mathrm{~N}^{-1}$ $+30 \mathrm{~kg} \mathrm{P}_{2} \mathrm{O}_{5} \mathrm{ha}^{-1}+10 \mathrm{~kg} \mathrm{~K}_{2} \mathrm{O} \mathrm{ha}^{-1}(18.15$ $\mathrm{cm})\left(\mathrm{T}_{11}\right)$.

The grain yield $\left(\mathrm{kg} \mathrm{ha}^{-1}\right)$ of treatment $\left(\mathrm{T}_{12}\right)$ i.e., application of $60 \mathrm{~kg} \mathrm{~N}^{-1}+30 \mathrm{~kg} \mathrm{P}_{2} \mathrm{O}_{5}$ $\mathrm{ha}^{-1}+20 \mathrm{~kg} \mathrm{~K}_{2} \mathrm{O} \mathrm{ha}^{-1}\left(1295 \mathrm{~kg} \mathrm{ha}^{-1}\right)$ was a significantly higher compared to application of $40 \mathrm{~kg} \mathrm{~N} \mathrm{ha}^{-1}+20 \mathrm{~kg} \mathrm{P}_{2} \mathrm{O}_{5} \mathrm{ha}^{-1}(903 \mathrm{~kg} \mathrm{ha}$ $\left.{ }^{1}\right)\left(\mathrm{T}_{13}\right)$ and control $\left(762 \mathrm{~kg} \mathrm{ha}^{-1}\right)\left(\mathrm{T}_{14}\right)$.

The straw yield $\left(\mathrm{kg} \mathrm{ha}^{-1}\right)$ of treatment $\left(\mathrm{T}_{12}\right)$ i.e., application of $60 \mathrm{~kg} \mathrm{~N}^{-1}+30 \mathrm{~kg} \mathrm{P}_{2} \mathrm{O}_{5}$ $\mathrm{ha}^{-1}+20 \mathrm{~kg} \mathrm{~K}_{2} \mathrm{O} \mathrm{ha}^{-1}\left(3131 \mathrm{~kg} \mathrm{ha}^{-1}\right)$ higher compared to other applicational though it was found on par with application of $60 \mathrm{~kg} \mathrm{~N} \mathrm{ha}^{-1}$ $+30 \mathrm{~kg} \mathrm{P}_{2} \mathrm{O}_{5} \mathrm{ha}^{-1}+10 \mathrm{~kg} \mathrm{~K}_{2} \mathrm{O} \mathrm{ha}^{-1}(2799 \mathrm{~kg}$ $\left.\mathrm{ha}^{-1}\right)\left(\mathrm{T}_{11}\right)$ but these two were found significantly higher as compared to other treatments and the control has recorded significantly the lower straw yield $(1682 \mathrm{~kg}$ $\left.h^{-1}\right)\left(T_{14}\right)$.

The increased in productive tillers, panicle length, grain yield and straw yield was due to interaction effects of nitrogen, phosphorous and potassium, which resulted in higher plant height, leaf area, total dry matter accumulation in plant, and its accumulation in different plant parts like leaf, stem and higher number of tillers. These observations are in line with observation made by Vimalan et al., (2019), Bhomte et al., (2016), Maitra et al., (2001) and Rakesh et al., (2015).

In conclusion the application of $60 \mathrm{~kg} \mathrm{~N}^{-1}$ $+30 \mathrm{~kg} \mathrm{P}_{2} \mathrm{O}_{5} \mathrm{ha}^{-1}+20 \mathrm{~kg} \mathrm{~K}_{2} \mathrm{O} \mathrm{ha}^{-1}$ has shown higher plant height, leaf area, dry matter accumulation, productive tillers, ear head length, grain yield and straw yield in Browntop millet.

\section{References}

Bhomte, M. V., Apotikar, V. A., and Pachpole, D. S. 2016. Effect of different fertilizer levels on growth and yield of little millet (Panicum sumantrense) genotypes. Contem. Res. Ind., 6(3),2231-2137.

Gomez, K. A., and Gomez, A. A. 1984. Statistical Procedures for Agric. Res. 2nd Ed. John Wiley \& sons, New York.

Lingegowda, B. K., Ashok, E. G., and Chandrappa, M. G. 1986. Investigation efficiency of nitrogen small millets. Mysore J. Agric. Sci.,11, 486 - 488.

Maitra, S., Ghosh, D. C., Sounda, G., and Jana, P. K. 2001. Performance of intercropping legumes in fingermillet (Eleusine coracana) at varying fertility levels. Indian J. Agron.,46(1),38-44.

Neha, G. G., Preeti, C., and Shobha, K. 2017. Effect of different nitrogen levels and varietal performance on growth and yield of summer pearl millet. Int. $J$. Curr. Microbiol. App. Sci.,6(6),861869.

Nigade, R. D., and More, S. M. 2012. Performance of finger millet varieties to different levels of fertilizer on yield and soil properties in sub-montane zone of Maharashtra. Int. J. Agri. Sci., 9(1),256259. 
Patil,S. V., Bhosale, A. S., and Khambal, P. D. 2015. Effect of various levels of fertilizers on growth and yield of finger millet. J. Agric. Veter. Sci.,8(6),49-52.

Rakesh,C., Yadav, L. R., and Parihar, S. 2015. Effect of vermicompost and fertility levels on growth and yield of pearl millet (Pennisetum glaucumL.). Ann. Arid Zone,54(1\&2),59-61.
Vimalan, B., Thiyageshwari, S., Balakrishnan, K., Rathinasamy, A., and Kumutha, K. 2019.Influence of NPK fertilizers on yield and uptake of barnyard millet grain (Echinochloa frumentacea (Roxb.) Link) in Typic Rhodulstalf soil. J. Pharmacology and Phytochemistry. 8(2), 1164-1166.

\section{How to cite this article:}

Kiran, H. P. 2021. Effect of Different Nutrient Levels on Growth and Yield of Browntop Millet. Int.J.Curr.Microbiol.App.Sci. 10(01): 3250-3254.

doi: https://doi.org/10.20546/ijcmas.2021.1001.378 